OPEN ACCESS

Edited by:

Witali L. Dunin-Barkowski, Russian Academy of Sciences (RAS), Russia

Reviewed by: Ivan Yurievich Tyukin, University of Leicester United Kingdom Danil Prokhorov

Toyota R \& D, Ann Arbor, United States

${ }^{*}$ Correspondence: Andrei Zinovyev andrei.zinovyev@curie.fr

Received: 31 October 2019 Accepted: 09 December 2019

Published: 09 January 2020

Citation:

Bac J and Zinovyev A (2020) Lizard Brain: Tackling Locally Low-Dimensional Yet Globally Complex Organization of

Multi-Dimensional Datasets.

Front. Neurorobot. 13:110. doi: 10.3389/fnbot.2019.00110

\section{Lizard Brain: Tackling Locally Low-Dimensional Yet Globally Complex Organization of Multi-Dimensional Datasets}

\author{
Jonathan Bac ${ }^{1,2,3,4}$ and Andrei Zinovyev ${ }^{1,2,3,5 *}$ \\ ${ }^{1}$ Institut Curie, PSL Research University, Paris, France, ${ }^{2}$ Institut National de la Santé et de la Recherche Médicale, U900, \\ Paris, France, ${ }^{3}$ MINES ParisTech, CBIO-Centre for Computational Biology, PSL Research University, Paris, France, ${ }^{4}$ Centre \\ de Recherches Interdisciplinaires, Université de Paris, Paris, France, ${ }^{5}$ Lobachevsky University, Nizhny Novgorod, Russia
}

Machine learning deals with datasets characterized by high dimensionality. However, in many cases, the intrinsic dimensionality of the datasets is surprisingly low. For example, the dimensionality of a robot's perception space can be large and multi-modal but its variables can have more or less complex non-linear interdependencies. Thus multidimensional data point clouds can be effectively located in the vicinity of principal varieties possessing locally small dimensionality, but having a globally complicated organization which is sometimes difficult to represent with regular mathematical objects (such as manifolds). We review modern machine learning approaches for extracting low-dimensional geometries from multi-dimensional data and their applications in various scientific fields.

Keywords: intrinsic dimension, dimension reduction, high-dimensional data, manifold learning, multi-manifold learning

\section{INTRODUCTION : HIGH-DIMENSIONAL BRAIN vS. LIZARD BRAIN IN HIGH-DIMENSIONAL WORLD}

The space of robotic perception or human-robot-control interfaces formed by features extracted from raw sensor measurements (including self-perception recorded, for example, by force/torque sensors, and perception of other active players such as humans) is high-dimensional (multi-modal) and can be characterized by non-trivial geometry and topology (Artemiadis and Kyriakopoulos, 2010; Droniou et al., 2015). Planning and taking decisions requires active unsupervised learning of perception space structure and, if necessary, correction of the learnt models on the fly without destroying accumulated experience (Li et al., 2019). This might require the emergence of specialized functions in the robot "brain."

Tackling the complexity of high-dimensional data spaces is a central challenge in machine learning. The famous notion of curse of dimensionality recapitulates difficulties with treating highdimensional datasets, related to the mathematical theory of measure concentration (Giannopoulos and Milman, 2000; Gromov, 2003). In machine learning, among other manifestations it can refer to a distance measure's loss of discriminatory power as the intrinsic dimension of data increases, due to a concentration of pairwise distances between points toward the same mean value. In this setting, machine learning approaches which rely on the notion of neighboring data points perform badly. In practical applications, treating high-dimensional data can be challenging in terms of computational and memory demands. On the other hand, the curse can also be a blessing: essentially high-dimensional data point clouds possess surprisingly simple organization, which has been recently exploited in the framework of high-dimensional brain in high-dimensional 
world (Gorban et al., 2019b). High-dimensional brain is a model for the codification of memories composed from many sparsely connected neurons, each of which only deals with few highdimensional data points, separating them from the rest of the data point cloud (Gorban et al., 2019b). It was applied to construct highly efficient error correctors of legacy AI systems, using non-iterative learning (Gorban et al., 2018).

The majority of unsupervised machine learning methods aim at reducing data's dimensionality or decomposing it into low-dimensional factors. This is opposite to the task of the high-dimensional brain, so we will call by analogy lizard brain a learning algorithm which is able to extract a useful lowdimensional representation of a high-dimensional data point cloud. Matching the level of data complexity, this representation can be complex and characterized by such features as nonlinearity, discontinuity (e.g., coarse-grained clusters or other types of deviation from sampling independence and uniformity), bifurcations, non-trivial topologies and varying local intrinsic dimension (ID). By usefulness we mean that the extracted representation would improve downstream learning tasks; for example, by modifying point neighborhood relations and data space metrics. The name lizard brain is inspired by the triune brain theory, stating the existence of several layered mammalian brain substructures sequentially evolved and specialized in different types of animal behaviors (MacLean, 1990). We do not claim that the real reptilian brain or the reptilian complex is of low-dimensional nature: here we use this metaphor only to underline that an effective learning system should be composed of several parts, built on top of each other and dealing with opposite aspects of the high-dimensional world.

Distinct tasks of lizard and high-dimensional brains in machine learning reflect the complementarity principle (Gorban and Tyukin, 2018; Gorban et al., 2019a): the data space can be split into a low volume (low dimensional) subset, which requires nonlinear methods for constructing complex data approximators, and a high-dimensional subset, characterized by measure concentration, and simplicity allowing the effective application of linear methods. Machine learning methodology should suggest a method for making such splitting in reallife datasets, and propose tools specialized in dealing with intrinsically low- and high-dimensional data parts.

In this short review, we focus on methods for quantifying intrinsic dimensionality and constructing useful summaries of the data, by projection into low-dimensional space, or projection onto principal geometrical objects of lower complexity that approximate the structure of the data point cloud. We introduce a classification of these methods based on the notions of mathematical projection theory.

\section{DEFINING AND MEASURING INTRINSIC DIMENSION}

The notion of intrinsic dimension (ID) intuitively refers to the minimal number of variables needed to represent data with little information loss. This concept, introduced in the field of signal analysis (Bennett, 1969), is largely used but doesn't have a consensus mathematical definition (Campadelli et al., 2015). In the context of the manifold hypothesis, i.e., when the data are considered to be a sample from an underlying $n$-dimensional manifold, the goal of ID estimation is to recover $n$.

Methods for ID estimation can be grouped by operating principle (Campadelli et al., 2015). The correlation dimension is an example of fractal method based on the fact that the number of points contained in a ball of growing radius $r$ scales exponentially with the dimension of the underlying manifold (Grassberger and Procaccia, 1983). Topological methods estimate the topological dimension (e.g., as defined by the Lebesgue covering dimension) of a manifold. Projective methods look at the effect of mapping the points onto a lower-dimensional subspace, and set a threshold dimension based on a cost function and various heuristics (e.g., looking at variance gaps in the eigenspectra) (Fukunaga and Olsen, 1971; Bruske and Sommer, 1998; Little et al., 2009b; Fan et al., 2010). Graph-based methods exploit scaling properties of graphs, such as the length of the minimum spanning tree (Costa and Hero, 2004). Nearest neighbors methods rely on scaling properties of the distribution of local distances or angles, due for example to measure concentration (Levina and Bickel, 2004; Ceruti et al., 2014; Johnsson, 2016; Facco et al., 2017; Wissel, 2018; Amsaleg et al., 2019; Díaz et al., 2019; Gomtsyan et al., 2019). It has also been recently proposed to use the Fisher separability statistic (i.e., the probability of a data point to be separated from the rest of the data point cloud by a Fisher discriminant) for the estimation of ID (Gorban and Tyukin, 2018; Albergante et al., 2019). The observed distribution is compared in terms of this statistic to the one expected for i.i.d. samples from a uniform distribution of given dimension to find the one with closest properties (e.g., the distribution of the "equivalent sphere").

Many ID estimators provide a single global ID value for the whole dataset but can be adapted to the case of varying local dimensionality by estimating the ID in data neighborhoods. The data contained in each neighborhood is usually assumed to be uniformly distributed over an $n$-dimensional ball (Levina and Bickel, 2004; Ceruti et al., 2014; Johnsson, 2016; Wissel, 2018; Díaz et al., 2019). In practice, ID proves sensitive to deviations from uniformity and neighborhood size (Little et al., 2009a; Campadelli et al., 2015). Benchmarks have shown that no single estimator today is ideal and using an ensemble of them is recommended (Campadelli et al., 2015; Camastra and Staiano, 2016).

\section{LEARNING LOW-DIMENSIONAL STRUCTURES OF HIGH-DIMENSIONAL DATA POINT CLOUDS}

The task of the lizard brain is to learn the low-dimensional structure of a data point cloud $x_{i}, i=1 \ldots m$, existing in highdimensional space $R^{N}$. The principal mathematical approach to solve this task consists in defining a map (projection) $\phi$ from $R^{N}$ to some base space $B$ which is characterized by intrinsic dimension smaller than $N$. The large variety of algorithms learning low-dimensional data structures can be grouped with respect to the details of $\phi$ implementation and the structure 
of $B$. If $B$ is Euclidean space $R^{k}, k<<N$ then the approach is usually related to the manifold learning framework (Ma and $\mathrm{Fu}, 2011)$. However, $B$ can be characterized by a more complex structure than simple Euclidean space: for example, it can have a non-trivial topology (of torus, sphere, dendroid, ...). The base space can be discontinuous, such as a set of principal points learnt by $K$-means clustering. The algorithm can learn the base space structure as in the elastic principal graph method (Gorban et al., 2008b) or in the Growing Self-Organizing Maps (GSOM) (Alahakoon et al., 2000). Sometimes, these approaches are also named manifold learning techniques even though what is learnt can be more complex than a simple single manifold.

Below we classify a method by whether it assumes the base space $B$ to be embedded or injected into the total space $R^{N}$. In this case, we call a method injective, otherwise it is classified as projective (only the projection function to the base space is learnt). In the injective case, the base space $B$ represents a subset of the initial data space $R^{N}$. Typically, in injective methods we assume that the injected $B$ is an approximation of data and use a nearest point for projection on $B$.

\subsection{Injective Methods With Simple Euclidean Base Space}

The classical method for extracting low-dimensional data structure is Principal Component Analysis (PCA) in which case $B$ is simply a linear manifold in $R^{N}, \phi$ is orthogonal projection onto $B$, and the sum of Euclidean distance squares $\left\|x_{i}-\phi\left(x_{i}\right)\right\|^{2}$ is minimized (Jolliffe, 1993). Some non-linear extensions of PCA such as Hastie's principal curves (Hastie, 1984) or the piece-wise linear principal curves (Kégl and Krzyzak, 2002) are also injective methods as well as the popular SelfOrganizing Map (SOM) (Kohonen, 1990). The SOM follows a stochastic approximation approach, while some of its descendant approaches optimize explicit functions: e.g., the Generative Topographic Map maximizes the likelihood of a low-dimensional Gaussian mixture distribution (Bishop et al., 1998), while the Elastic Map is based on optimization of the elastic energy functional (Gorban and Rossiev, 1999; Zinovyev, 2000; Gorban and Zinovyev, 2005, 2010; Gorban et al., 2008a), defined on a regular grid of nodes embedded into the data space. The Elastic Map approach can approximate data by manifolds with arbitrarily chosen topologies, e.g., by closed principal curves or spherical manifolds (Gorban and Zinovyev, 2005, 2009). For methods fitting a set of nodes to the data, the base space is either defined in the nodes of the grid or by linear interpolation between nodes: for example, a curve is defined as a set of nodes and linear segments connecting them, a 2D manifold is defined by triangulation of the grid and using linear segments, etc. The projection operator is frequently defined as a projection onto the nearest point of the manifold.

Currently we face a rapidly increasing interest in unsupervised learning methods based on artificial neural networks (ANNs). For example, the autoencoder ANNs, proposed in the early 90s, are trained to reproduce input data and are characterized by an hourglass organization, with a middle bottleneck layer containing few neurons and constraining the network to generate the output from a compressed input representation (Kramer, 1991; Hinton and Salakhutdinov, 2006). The base space is represented by the signals on the bottleneck layer neurons and usually is a simple Euclidean space. ANN-based autoencoders can be considered injective methods since any combination of signals at the bottleneck layer can be mapped back into the data space by the demapping ANN layers. Variational autoencoders learn in the bottleneck layer parameters of some intrinsically low-dimensional probabilistic graphical model generating the data (Kingma and Welling, 2013). Moreover, graph neural networks, including graph autoencoders, are able to perform dimensionality reduction by producing summarized graph-based embeddings of data (Scarselli et al., 2008), a feature related to the next section.

\subsection{Injective Methods With Base Space Having Complex Structure}

Injective methods with Euclidean base space help representing the intrinsic dataset complexity by reducing dimensionality but do not reflect this complexity in the structure of the base space. Other methods learn the structure of the base space such that it reflects that of the data point cloud. Initially (growing), neural gas algorithms used Hebbian learning to reconstruct summaries of data topology which can, however, remain too complex (Martinetz et al., 1991; Fritzke, 1995). The growing SOM derives regular base space structure which can have varying ID (Alahakoon et al., 2000).

Principal graphs together with methods for fitting them to data are a flexible framework for learning low-dimensional structures (Gorban and Zinovyev, 2010). In practice, the graph complexity should be constrained. For example, principal trees construct base spaces having dendroid topologies, which is achieved, in the Elastic Principal Graph (ElPiGraph) approach, by the application of topological grammar rules, transforming trees into trees and thus exploring only a space of trees (Gorban et al., 2007). A richer set of grammar rules can explore larger graph families (Albergante et al., 2018). Other methods are based on heuristics to guess the graph structure; for example, extracting the Minimal Spanning Tree from the kNN-graph in the Simple Principal Tree (simplePPT) method (Mao et al., 2015) automatically imposes the tree-like structure on the base space. Principal complexes combine the advantages of using regular grid (too restricted) and arbitrary graph (too complex) structures to approximate data. Here the graph grammar rules are applied to a small number of factor graphs, while the resulting structure of the approximating object is defined by the Cartesian product of factors (Gorban et al., 2007). For example, the Cartesian product of two linear graphs produces a $2 \mathrm{D}$ rectangular grid, and the Cartesian product of a tree-like graph with a linear graph will fit a branching sheet-like structure to the data. This approach allows constructing complex principal objects with ID larger than one controlling the complexity of graph factors only.

\subsection{Projective Methods}

In projective methods, the base space $B$ which can possess more or less complex internal structure is not assumed to be a subset of the total space $R^{N}$. This provides flexibility in the 
algorithm's construction but limits the capability for mapping new objects not participating in the definition of the projection (out-of-sample objects) from $R^{N}$ into $B$. In other words, the mapping is learnt only for a subset of points in $B$ corresponding to the data vectors $x_{i} \in R^{N}$ and not the rest of the data space. We note that the majority of projective methods start by computing an object similarity or dissimilarity matrix or offshoots of it, such as $k$-nearest neighbors $(\mathrm{kNN})$ graph or $\epsilon$ graph. The predecessor of many modern projective methods is the classical Multi-Dimensional Scaling (MDS) which is a linear projective alternative of PCA (Torgerson, 1952).

The most popular representatives of non-linear projective methods are ISOMAP (Tenenbaum et al., 2000), Laplacian and Hessian Eigenmaps (Belkin and Niyogi, 2003; Donoho and Grimes, 2003) and Diffusion maps (Coifman and Lafon, 2006), in which the main idea is to define object dissimilarity reflecting the geodesic distances along the $\mathrm{kNN}$ - or $\epsilon$-graph (see Figure 1). Local Linear Embedding (LLE) aims at reproducing, in the lowdimensional space, local linear relations between objects in the total space and assemble them into a global picture (Roweis and Saul, 2000; Zhang and Wang, 2007). Kernel PCA exploits the kernel trick and applies MDS on a kernel-modified Gram matrix (Schölkopf et al., 1998; Bengio et al., 2004a; Ham et al., 2004). On top of the original formulations, many generalizations of these methods have been produced recently. For example, the vector diffusion map (Singer and Wu, 2012) doesn't use operators on the manifold itself but differential operators on fiber bundles over the manifold. Grassmann\&Stiefel Eigenmaps require proximity between the original manifold and its estimator but also between their tangent spaces (Bernstein and Kuleshov, 2012; Bernstein et al., 2015). The limitations of the projective methods are partially overcome in some of their out-of-sample extensions that allow the mapping of new points without having to recompute eigenvectors (Bengio et al., 2004b; Qiao et al., 2012).

Several methods for projective dimensionality reduction, such as t-distributed stochastic neighboring embedding ( $\mathrm{t}$-SNE) (Maaten and Hinton, 2008) or more recent Uniform Manifold Approximation and Projection (UMAP) (McInnes et al., 2018) found overwhelming number of applications in applied data science, e.g., for visualizing large-scale molecular profiling data in biology. One of the reasons for their popularity is their focus on more accurate representation of small distances (rather than large ones as in PCA) between data vectors, which frequently match better the purpose of data visualization/representation.

Projective methods are extremely popular in modern machine learning for non-linear dimensionality reduction, and new ideas are constantly explored: here we can mention kernel density estimation (Mohammed and Narayanan, 2017), genetic programming (Lensen et al., 2019), parallel transport (Budninskiy et al., 2019), triplet information (TRIMAP) (Amid and Warmuth, 2019).

While the vast majority of methods use projection onto Euclidean base space, some authors have also suggested the use of classical algorithms for non-Euclidean embeddings, such as hyperbolic or spherical spaces (Begelfor and Werman, 2005; Cvetkovski and Crovella, 2017). Recently, several works have shown benefits of non-Euclidean embeddings for the particular case of graph data, which can have intrinsic curvature (Walter and Ritter, 2002; Chamberlain et al., 2017; Muscoloni et al., 2017; Nickel and Kiela, 2017).

\subsection{Multi-Manifold and Manifold Alignment Learning}

The complex and sometimes discontinuous organization of reallife data can be a challenge for the single manifold hypothesis, which underlies many algorithms. In some cases, data is better described as sampled from multiple manifolds. For example, the task of face recognition can be described by the identification of different manifolds, each corresponding to a different person's facial images (Yang et al., 2007). Another example is LIDAR technology, which generates $3 \mathrm{D}$ point clouds in the form of the surrounding terrain (e.g., a bridge will result in a flat 2D surface for the road, 1D cables, etc.) (Medina et al., 2019).

The existence of such data motivates approaches that account for the presence of multiple and potentially intersecting manifolds. A first idea to deal with such scenario is to measure local ID to identify structures with variable ID in a dataset. As a natural next step, the data can be segmented accordingly to the local ID (see Allegra et al., 2019 and references therein). Beyond such segmentation, one can integrate classical algorithms into a complete framework to perform the detection and reconstruction of manifold structures. Such frameworks have been recently introduced based on well-known algorithms, such as spectral clustering and local tangent space estimation (Wang et al., 2010, 2011; Gong et al., 2012), LLE (Hettiarachchi and Peters, 2015), ISOMAP (Fan et al., 2012; Yang et al., 2016; Li et al., 2017; Mahapatra and Chandola, 2017) and local PCA (Arias-Castro et al., 2017). Other approaches use less classical techniques such as tensor voting (Mordohai and Medioni, 2010; Deutsch and Medioni, 2015, 2016), variational autoencoders (Ye and Zhao, 2019), or multi-agent flow (Shen and Han, 2016).

Another task which becomes important in some scientific domains is to learn distinct maps from several data spaces to the common base space. The general idea here is to align, according to some criteria, multiple projections of the data point clouds; therefore, this family of methods is sometimes termed "manifold alignment" (Ma and Fu, 2011). Details of the problem formulation are important here and can constrain the method applicability. For example, Generalized Unsupervised Manifold Alignment (GUMA) assumes a possibility of one-toone mapping between two data spaces (Cui et al., 2014). The Manifold Alignment Generative Adversarial Network (MAGAN) uses generative adversarial networks (GAN) to use one data space as a base space for a second data space, and vice versa (Amodio and Krishnaswamy, 2018); it assumes either some shared variables or partly matched pairs of points between two data spaces.

\section{DISCUSSION}

In this short review we highlight that many globally multidimensional datasets used in the field of machine learning and artificial intelligence can possess intrinsically low-dimensional 


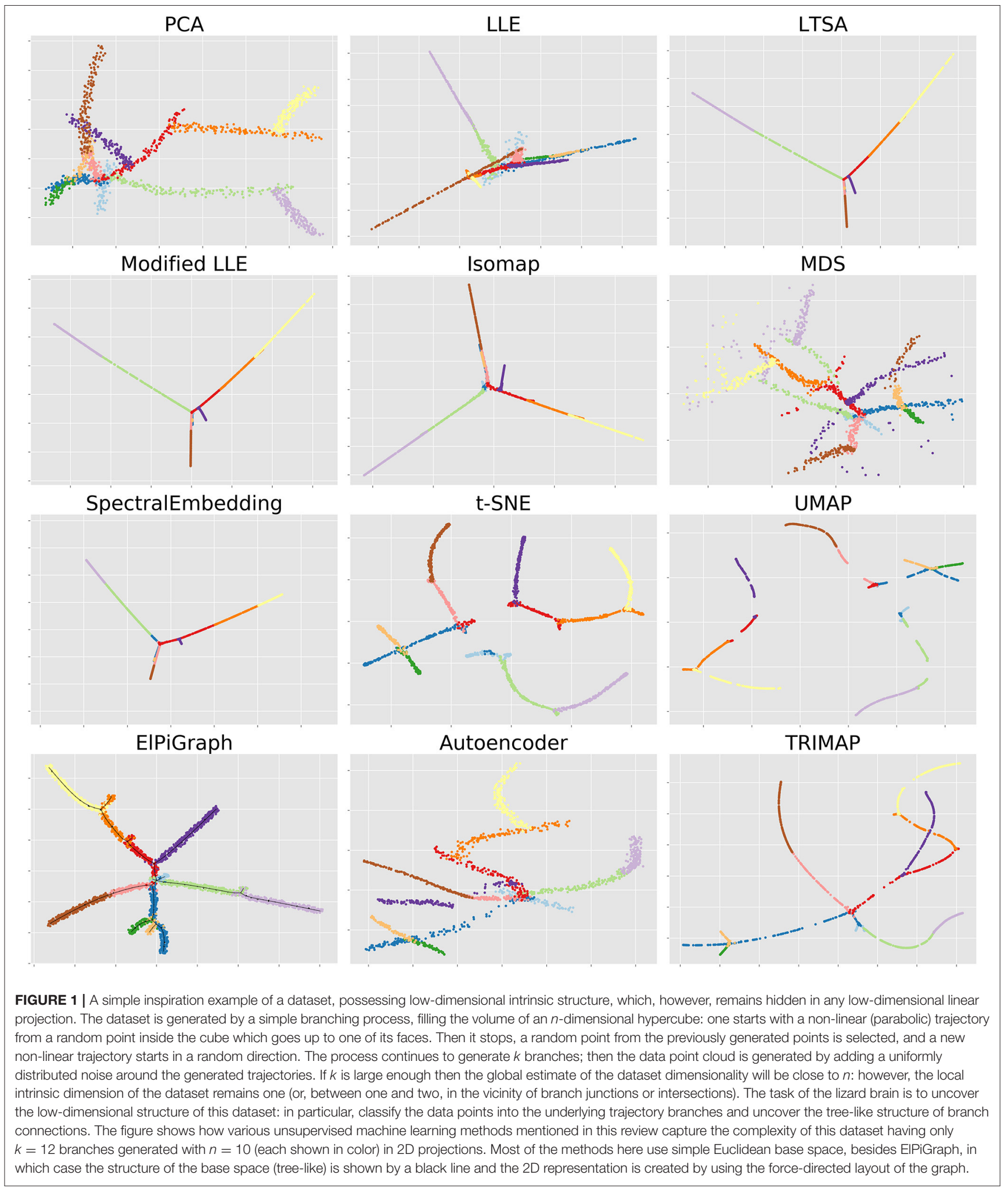

structure, which yet can be highly complex. The task of a lizard brain (methaphoric opposite to the high-dimensional brain, composed of sparsely connected concept neurons) is to detect which parts of the data are essentially low-dimensional and to extract the low-dimensional structure from high-dimensional space. Well-established manifold learning frameworks can 
be used for this purpose, taking into account some recent developments mentioned above. At the same time, new approaches learning structures more general than simple connected manifolds are needed in concrete applications. Thus, the structure of real-life datasets can be characterized by strong noise, bifurcation-like patterns, self-intersecting flows, variable local ID, fine-grained lumping, and other features not easily captured by the manifold-type objects. There exists candidate methodologies such as data approximation by principal cubic complexes, using topological grammar approach, which can overcome some limitations of the simple manifoldbased approaches.

There are scientific fields where the data possessing complex yet locally low-dimensional structure are generated at large scale. One example of this is molecular profiling of single cells in molecular biology, where the generated clouds of data points are characterized by many of the above mentioned complex features. Today we face a boom of machine learning-based methodology development aiming at treating this data type (Chen et al., 2019; Saelens et al., 2019). Another well-known example is reconstructing the surrounding environment from point clouds generated by LIDAR technology.

\section{REFERENCES}

Alahakoon, D., Halgamuge, S. K., and Srinivasan, B. (2000). Dynamic selforganizing maps with controlled growth for knowledge discovery. IEEE Trans. Neural Netw. 11, 601-614. doi: 10.1109/72.846732

Albergante, L., Bac, J., and Zinovyev, A. (2019). "Estimating the effective dimension of large biological datasets using fisher separability analysis," in Proceedings of the IEEE IJCNN 2019 - International Joint Conference on Neural Networks (Budapest: IEEE). doi: 10.1109/IJCNN.2019.88 52450

Albergante, L., Mirkes, E. M., Chen, H., Martin, A., Faure, L., Barillot, E., et al. (2018). Robust and scalable learning of complex dataset topologies via ElPiGraph. arXiv [preprint] arXiv:1804.07580.

Allegra, M., Facco, E., Laio, A., and Mira, A. (2019). Clustering by the local intrinsic dimension: the hidden structure of real-world data. arXiv [preprint] arXiv:1902.10459.

Amid, E., and Warmuth, M. K. (2019). Trimap: large-scale dimensionality reduction using triplets. arXiv [preprint] arXiv:1910.00204.

Amodio, M., and Krishnaswamy, S. (2018). MAGAN: aligning biological manifolds. arXiv [preprint] arXiv:1803.00385.

Amsaleg, L., Chelly, O., Houle, M. E., Kawarabayashi, K.-I., Radovanović, M., and Treeratanajaru, W. (2019). "Intrinsic dimensionality estimation within tight localities," in Proceedings of the 2019 SIAM International Conference on Data Mining (SIAM), 181-189.

Arias-Castro, E., Lerman, G., and Zhang, T. (2017). Spectral clustering based on local PCA. J. Mach. Learn. Res. 18, 253-309. Available online at: http://dl.acm. org/citation.cfm?id=3122009.3122018

Artemiadis, P. K., and Kyriakopoulos, K. J. (2010). EMG-based control of a robot arm using low-dimensional embeddings. IEEE Trans. Robot. 26, 393-398. doi: 10.1109/TRO.2009.2039378

Begelfor, E., and Werman, M. (2005). The world is not always flat or learning curved manifolds. School Eng. Comput. Sci. Hebrew Univer. Jerusalem. Tech. Rep. 3:8. Available online at: http://www.cs.huji.ac.il/ werman/Papers/cmds. pdf

Belkin, M., and Niyogi, P. (2003). Laplacian eigenmaps for dimensionality reduction and data representation. Neural Comput. 15, 1373-1396. doi: $10.1162 / 089976603321780317$
Further efforts are needed to supply the lizard brain with algorithmic approaches suitable in the various contexts of real-life data. The development of benchmark datasets and new benchmarking methodologies is also needed to assess the efficiency and applicability of the existing toolbox for extracting low-dimensional structures from high-dimensional data.

\section{AUTHOR CONTRIBUTIONS}

AZ and JB jointly defined the scope of the review, its bibliography and classification of methods, wrote the review and together worked on the implementation of the Jupyter notebook.

\section{FUNDING}

This work has been partially supported by the Ministry of Science and Higher Education of the Russian Federation (Project No. 14.Y26.31.0022) and the doctoral school Frontiéres de l'Innovation en Recherche et Education-Programme Bettencourt.

Bengio, Y., Delalleau, O., Roux, N. L., Paiement, J.-F., Vincent, P., and Ouimet, M. (2004a). Learning eigenfunctions links spectral embedding and kernel PCA. Neural Comput. (Montreal, QC) 16, 2197-2219. doi: 10.1162/0899766041732396

Bengio, Y., Paiement, J.-F., Vincent, P., Delalleau, O., Roux, N. L., and Ouimet, M. (2004b). "Out-of-sample extensions for LLE, ISOMAP, MDS, eigenmaps, and spectral clustering," in Advances in Neural Information Processing Systems, $177-184$.

Bennett, R. (1969). The intrinsic dimensionality of signal collections. IEEE Trans. Inform. Theory 15, 517-525.

Bernstein, A., Kuleshov, A., and Yanovich, Y. (2015). "Information preserving and locally isometric\&conformal embedding via tangent manifold learning," in 2015 IEEE International Conference on Data Science and Advanced Analytics (DSAA) (Paris: IEEE), 1-9. doi: 10.1109/DSAA.2015.73 44815

Bernstein, A. V., and Kuleshov, A. P. (2012). Tangent bundle manifold learning via Grassmann\&Stiefel eigenmaps. arXiv [preprint] arXiv:1212.6031.

Bishop, C. M., Svensén, M., and Williams, C. K. (1998). GTM: the generative topographic mapping. Neural Comput. 10, 215-234.

Bruske, J., and Sommer, G. (1998). Intrinsic dimensionality estimation with optimally topology preserving maps. IEEE Trans. Pattern Analy. Mach. Intell. 20, 572-575. doi: 10.1109/34.682189

Budninskiy, M., Yin, G., Feng, L., Tong, Y., and Desbrun, M. (2019). Parallel transport unfolding: a connection-based manifold learning approach. SIAM J. Appl. Algebra Geomet. 3, 266-291. doi: 10.1137/18M11 96133

Camastra, F., and Staiano, A. (2016). Intrinsic dimension estimation: advances and open problems. Inform. Sci. 328, 26-41. doi: 10.1016/j.ins.2015.08.029

Campadelli, P., Casiraghi, E., Ceruti, C., and Rozza, A. (2015). Intrinsic dimension estimation: relevant techniques and a benchmark framework. Math. Prob. Eng. 2015, 1-21. doi: 10.1155/2015/759567

Ceruti, C., Bassis, S., Rozza, A., Lombardi, G., Casiraghi, E., and Campadelli, P. (2014). DANCo: An intrinsic dimensionality estimator exploiting angle and norm concentration. Pattern Recognit. 47, 2569-2581. doi: 10.1016/j.patcog.2014.02.013

Chamberlain, B. P., Clough, J., and Deisenroth, M. P. (2017). Neural embeddings of graphs in hyperbolic space. arXiv [preprint] arXiv:1705.10359. 
Chen, H., Albergante, L., Hsu, J. Y., Lareau, C. A., Lo Bosco, G., Guan, J., et al. (2019). Single-cell trajectories reconstruction, exploration and mapping of omics data with STREAM. Nat. Commun. 10:1903. doi: 10.1038/s41467-019-09670-4

Coifman, R. R., and Lafon, S. (2006). Diffusion maps. Appl. Comput. Harmon. Analy. 21, 5-30. doi: 10.1016/j.acha.2006.04.006

Costa, J. A., and Hero, A. O. (2004). Geodesic entropic graphs for dimension and entropy estimation in manifold learning. IEEE Trans. Signal Process. 52, 2210-2221. doi: 10.1109/TSP.2004.831130

Cui, Z., Chang, H., Shan, S., and Chen, X. (2014). "Generalized unsupervised manifold alignment," in Advances in Neural Information Processing Systems, (Montreal, QC) 2429-2437.

Cvetkovski, A., and Crovella, M. (2017). Low-stress data embedding in the hyperbolic plane using multidimensional scaling. Appl. Math. 11, 5-12. doi: $10.18576 / \mathrm{amis} / 110102$

Deutsch, S., and Medioni, G. (2016). Learning the geometric structure of manifolds with singularities using the tensor voting graph. J. Math. Imaging. Vision 57, 402-422. doi: 10.1007/s10851-016-0684-2

Deutsch, S., and Medioni, G. G. (2015). "Intersecting manifolds: detection, segmentation, and labeling," in Twenty-Fourth International Joint Conference on Artificial Intelligence, (Buenos Aires) 3445-3452.

Díaz, M., Quiroz, A. J., and Velasco, M. (2019). Local angles and dimension estimation from data on manifolds. J. Multivar. Analy. 173, 229-247. doi: 10.1016/j.jmva.2019.02.014

Donoho, D. L., and Grimes, C. (2003). Hessian eigenmaps: locally linear embedding techniques for high-dimensional data. Proc. Natl. Acad. Sci. U.S.A. 100, 5591-5596. doi: 10.1073/pnas. 1031596100

Droniou, A., Ivaldi, S., and Sigaud, O. (2015). Deep unsupervised network for multimodal perception, representation and classification. Robot. Autonomous Syst. 71, 83-98. doi: 10.1016/j.robot.2014.11.005

Facco, E., D'Errico, M., Rodriguez, A., and Laio, A. (2017). Estimating the intrinsic dimension of datasets by a minimal neighborhood information. Sci. Rep. 7:12140. doi: 10.1038/s41598-017-11873-y

Fan, M., Gu, N., Qiao, H., and Zhang, B. (2010). Intrinsic dimension estimation of data by principal component analysis. arXiv 1002.2050 [cs.CV].

Fan, M., Qiao, H., Zhang, B., and Zhang, X. (2012). "Isometric multimanifold learning for feature extraction" in 2012 IEEE 12th International Conference on Data Mining (Brussels: IEEE) 241-250. doi: 10.1109/ICDM.2 012.98

Fritzke, B. (1995). "A growing neural gas network learns topologies," in Advances in Neural Information Processing Systems, (Denver, CO) 625-632.

Fukunaga, K., and Olsen, D. (1971). An algorithm for finding intrinsic dimensionality of data. IEEE Trans. Comput. C-20, 176-183.

Giannopoulos, A., and Milman, V. (2000). Concentration property on probability spaces. Adv. Math. 156, 77-106. doi: 10.1006/aima.2000.1949

Gomtsyan, M., Mokrov, N., Panov, M., and Yanovich, Y. (2019). Geometryaware maximum likelihood estimation of intrinsic dimension. arXiv [preprint] arXiv:1904.06151.

Gong, D., Zhao, X., and Medioni, G. (2012). Robust multiple manifolds structure learning. arXiv [preprint] arXiv:1206.4624.

Gorban, A., Golubkov, A., Grechuk, B., Mirkes, E., and Tyukin, I. (2018). Correction of AI systems by linear discriminants: probabilistic foundations. Inf. Sci. (New York, NY) 466, 303-322. doi: 10.1016/j.ins.2018.07.040

Gorban, A., Kégl, B., Wunsch, D., and Zinovyev, A., editors (2008a). Principal Manifolds for Data Visualisation and Dimension Reduction. Berlin; Heidelberg; New York, NY: Springer.

Gorban, A., Makarov, V., and Tyukin, I. (2019a). Symphony of high-dimensional brain. Reply to comments on "The unreasonable effectiveness of small neural ensembles in high-dimensional brain". Phys. Life Rev. 29, 115-119. doi: 10.1016/j.plrev.2019.06.003

Gorban, A., Makarov, V., and Tyukin, I. (2019b). The unreasonable effectiveness of small neural ensembles in high-dimensional brain. Phys. Life Rev. 29, 55-88. doi: 10.1016/j.plrev.2018.09.005

Gorban, A., and Rossiev A. A. (1999). Neural network iterative method of principal curves for data with gaps. J. Comput. Syst. Sci. Int. 38, 825-830.

Gorban, A., and Tyukin, I. (2018). Blessing of dimensionality: mathematical foundations of the statistical physics of data. Phil. Trans. R. Soc. A 376:20170237. doi: 10.1098/rsta.2017.0237
Gorban, A., and Zinovyev, A. (2005). Elastic principal graphs and manifolds and their practical applications. Computing 75, 359-379. doi: 10.1007/s00607-005-0122-6

Gorban, A., and Zinovyev, A. (2010). Principal manifolds and graphs in practice: from molecular biology to dynamical systems. Int. J. Neural Syst. 20, 219-232. doi: $10.1142 / S 0129065710002383$

Gorban, A. N., Sumner, N. R., and Zinovyev, A. Y. (2007). Topological grammars for data approximation. Appl. Math. Lett. 20, 382-386. doi: 10.1016/j.aml.2006.04.022

Gorban, A. N., Sumner, N. R., and Zinovyev, A. Y. (2008b). "Beyond the concept of manifolds: Principal trees, metro maps, and elastic cubic complexes," in Principal Manifolds for Data Visualization and Dimension Reduction (Springer), 219-237.

Gorban, A. N., and Zinovyev, A. (2009). "Principal graphs and manifolds," in Handbook of Research on Machine Learning Applications and Trends: Algorithms, Methods and Techniques, eds E. S. Olivas, J. D. M. Guererro, M. M. Sober, J. R. M. Benedito, Lopes A.J.S.

Grassberger, P., and Procaccia, I. (1983). Measuring the strangeness of strange attractors. Phys. D Nonlinear Phenom. 9, 189-208.

Gromov, M. (2003). Isoperimetry of waists and concentration of maps. Geom. Funct. Anal. 13, 178-215. doi: 10.1007/s000390300004

Ham, J. H., Lee, D. D., Mika, S., and Schölkopf, B. (2004). A kernel view of the dimensionality reduction of manifolds. Departmental Papers (ESE), (Philadelphia, PA) 93.

Hastie, T. (1984). Principal curves and surfaces. Technical report, Stanford university, CA, Lab for computational statistics.

Hettiarachchi, R., and Peters, J. F. (2015). Multi-manifold LLE learning in pattern recognition. Patt. Recognit. 48, 2947-2960. doi: 10.1016/j.patcog.2015.04.003

Hinton, G. E., and Salakhutdinov, R. R. (2006). Reducing the dimensionality of data with neural networks. Science 313, 504-507. doi: 10.1126/science.1127647

Johnsson, K. (2016). Structures in High-Dimensional Data: Intrinsic Dimension and Cluster Analysis. Ph.D. thesis, Faculty of Engineering, LTH.

Jolliffe, I. (1993). Principal Component Analysis. Berlin; Heidelberg: Springer.

Kégl, B., and Krzyzak, A. (2002). Piecewise linear skeletonization using principal curves. IEEE Trans. Patt. Analy. Mach. Intell. 24, 59-74. doi: 10.1109/34.982884

Kingma, D. P., and Welling, M. (2013). Auto-encoding variational bayes. arXiv [preprint] arXiv:1312.6114.

Kohonen, T. (1990). The self-organizing map. Proc. IEEE 78, 1464-1480.

Kramer, M. A. (1991). Nonlinear principal component analysis using autoassociative neural networks. AIChE J. 37, 233-243.

Lensen, A., Xue, B., and Zhang, M. (2019). "Can genetic programming do manifold learning too?” in European Conference on Genetic Programming (Leipzig: Springer), 114-130

Levina, E., and Bickel, P. J. (2004). "Maximum Likelihood estimation of intrinsic dimension," in Proceedings of the 17th International Conference on Neural Information Processing Systems (Vancouver, BC: MIT Press), 777-784.

Li, X., Cai, C., and He, J. (2017). "Density-based multi-manifold ISOMAP for data classification," in 2017 Asia-Pacific Signal and Information Processing Association Annual Summit and Conference (APSIPA ASC) (Kuala Lumpur: IEEE), 897-903.

Li, Z., Chen, F., Bicchi, A., Sun, Y., and Fukuda, T. (2019). Guest Editorial Neuro-Robotics Systems: Sensing, Cognition, Learning, and Control. IEEE Trans. Cogn. Dev. Syst. 11, 145-147. doi: 10.1109/TCDS.2019.29 15408

Little, A. V., Jung, Y.-M., and Maggioni, M. (2009a). Multiscale Estimation of Intrinsic Dimensionality of Data Sets. Technical report. Arlington, TX, United States.

Little, A. V., Lee, J., Jung, Y.-M., and Maggioni, M. (2009b). "Estimation of intrinsic dimensionality of samples from noisy low-dimensional manifolds in high dimensions with multiscale SVD," in 2009 IEEE/SP 15th Workshop on Statistical Signal Processing (Cardiff: IEEE) 85-88.

Ma, Y., and Fu, Y. (2011). Manifold Learning Theory and Applications. Boca Raton, FL: CRC press.

Maaten, L. v. d., and Hinton, G. (2008). Visualizing data using t-SNE. J. Mach. Learn. Res. 9, 2579-2605. Available online at: http://www.jmlr.org/papers/v9/ vandermaaten $08 \mathrm{a} . \mathrm{html}$

MacLean, P. D. (1990). The Triune Brain in Evolution. New York, NY: Plenum. 
Mahapatra, S., and Chandola, V. (2017). "S-isomap++: multi manifold learning from streaming data," in IEEE International Conference on Big Data (Big Data) (Boston, MA: IEEE), 716-725.

Mao, Q., Yang, L., Wang, L., Goodison, S., and Sun, Y. (2015). "SimplePPT: a simple principal tree algorithm," in Proceedings of the 2015 SIAM International Conference on Data Mining (Vancouver, BC: SIAM), 792-800.

Martinetz, T., Schulten, K., et al. (1991). A "neural-gas" Network Learns Topologies. Champaign, IL: University of Illinois at Urbana-Champaign.

McInnes, L., Healy, J., and Melville, J. (2018). UMAP: Uniform manifold approximation and projection for dimension reduction. arXiv [preprint] arXiv:1802.03426.

Medina, F. P., Ness, L., Weber, M., and Djima, K. Y. (2019). "Heuristic framework for multiscale testing of the multi-manifold hypothesis," in Research in Data Science, eds E. Gasparovic and C. Domeniconi (Providence, RI: Springer), 47-80.

Mohammed, K., and Narayanan, H. (2017). Manifold learning using kernel density estimation and local principal components analysis. arXiv [preprint] arXiv: 1709.03615 .

Mordohai, P., and Medioni, G. (2010). Dimensionality estimation, manifold learning and function approximation using tensor voting. J. Mach. Learn. Res. 11, 411-450.

Muscoloni, A., Thomas, J. M., Ciucci, S., Bianconi, G., and Cannistraci, C. V. (2017). Machine learning meets complex networks via coalescent embedding in the hyperbolic space. Nat. Commun. 8:1615. doi: 10.1038/s41467-01701825-5

Nickel, M., and Kiela, D. (2017). "Poincaré embeddings for learning hierarchical representations," in Advances in Neural Information Processing Systems, (Long Beach, CA) 6338-6347.

Qiao, H., Zhang, P., Wang, D., and Zhang, B. (2012). An explicit nonlinear mapping for manifold learning. IEEE Trans. Cybernet. 43, 51-63. doi: 10.1109/TSMCB.2012.2198916

Roweis, S. T., and Saul, L. K. (2000). Nonlinear dimensionality reduction by locally linear embedding. Science 290, 2323-2326. doi: 10.1126/science.290.5500. 2323

Saelens, W., Cannoodt, R., Todorov, H., and Saeys, Y. (2019). A comparison of single-cell trajectory inference methods. Nat. Biotechnol. 37, 547-554. doi: 10.1038/s41587-019-0071-9

Scarselli, F., Gori, M., Tsoi, A. C., Hagenbuchner, M., and Monfardini, G. (2008). The graph neural network model. IEEE Trans. Neural Netw. 20, 61-80. doi: 10.1109/TNN.2008.2005605

Schölkopf, B., Smola, A., and Müller, K.-R. (1998). Nonlinear component analysis as a kernel eigenvalue problem. Neural Comput. 10, 1299-1319.

Shen, G., and Han, D. (2016). "A flow based approach for learning multiple manifolds," in 9th International Congress on Image and Signal Processing, BioMedical Engineering and Informatics (CISP-BMEI) (Datong: IEEE), 1905-1910.
Singer, A., and Wu, H.-T. (2012). Vector diffusion maps and the connection Laplacian. Commun. Pure Appl. Mathemat. 65, 1067-1144. doi: 10.1002/cpa.21395

Tenenbaum, J. B., Silva, V. d., and Langford, J. C. (2000). A global geometric framework for nonlinear dimensionality reduction. Science 290, 2319-2323. doi: $10.1126 /$ science.290.5500.2319

Torgerson, W. S. (1952). Multidimensional scaling: I. theory and method. Psychometrika 17, 401-419.

Walter, J. A., and Ritter, H. (2002). "On interactive visualization of highdimensional data using the hyperbolic plane," in Proceedings of the Eighth ACM SIGKDD International Conference on Knowledge Discovery and Data Mining (New York, NY: ACM), 123-132.

Wang, Y., Jiang, Y., Wu, Y., and Zhou, Z.-H. (2010). "Multi-manifold clustering," in Pacific Rim International Conference on Artificial Intelligence, (Daegu) 280291.

Wang, Y., Jiang, Y., Wu, Y., and Zhou, Z.-H. (2011). Spectral clustering on multiple manifolds. IEEE Trans. Neural Netw. 22, 1149-1161. doi: 10.1109/TNN.2011.2147798

Wissel, D. R. (2018). Intrinsic Dimension Estimation using Simplex Volumes. Ph.D. thesis, University of Bonn, Bonn, Germany.

Yang, B., Xiang, M., and Zhang, Y. (2016). Multi-manifold discriminant Isomap for visualization and classification. Patt. Recog. 55, 215-230. doi: 10.1016/j.patcog.2016.02.001

Yang, J., Zhang, D., Yang, J.-y., and Niu, B. (2007). Globally maximizing, locally minimizing: unsupervised discriminant projection with applications to face and palm biometrics. IEEE Trans. Patt. Analy. Mach. Intell. 29, 650-664. doi: 10.1109/TPAMI.2007.1008

Ye, X., and Zhao, J. (2019). Multi-manifold clustering: a graphconstrained deep nonparametric method. Patt. Recogn. 93, 215-227. doi: 10.1016/j.patcog.2019.04.029

Zhang, Z., and Wang, J. (2007). "MLLE: modified locally linear embedding using multiple weights," in Advances in Neural Information Processing Systems, (Vancouver, BC) 1593-1600.

Zinovyev, A. (2000). Visualization of Multidimensional Data [in Russian]. Krasnoyarsk: Krasnoyarsk State Technical University Press.

Conflict of Interest: The authors declare that the research was conducted in the absence of any commercial or financial relationships that could be construed as a potential conflict of interest.

Copyright (c) $2020 \mathrm{Bac}$ and Zinovyev. This is an open-access article distributed under the terms of the Creative Commons Attribution License (CC BY). The use, distribution or reproduction in other forums is permitted, provided the original author(s) and the copyright owner(s) are credited and that the original publication in this journal is cited, in accordance with accepted academic practice. No use, distribution or reproduction is permitted which does not comply with these terms. 